\title{
Contributions by Dr. Walter C. McCrone to the Field of Thermomicroscopy
}

John A. Smoliga, Ph.D.

Boehringer Ingelheim Pharmaceuticals, Inc. 900 Ridgebury Rd., Ridgefield, CT 06877

Thermomicroscopy is the analysis of samples involving the combination of controlled temperature with polarized light microscopy. Dr. Walter McCrone pioneered various techniques within this field which continue to provide unique information pertaining to the physical characterization of solid substances. These techniques can involve the use of apparatus as simple as a sample mounted on a microscope slide held over a microflame, to sophisticated equipment capable of spanning the temperature range between $-200^{\circ} \mathrm{C}$ to $+2500^{\circ} \mathrm{C}$. Data pertaining to substance purity, melting point, phase transitions, relative stability of polymorphs, solvation state, kinetics of crystal growth and analysis of mixtures are among the various applications of thermomicroscopy ${ }^{1}$. While widely applicable to the general area of industrial microscopy, thermomicroscopy is particularly useful in the pharmaceutical industry for solid state characterization of drug substances and drug products. This paper will review Walter McCrones' contributions to thermomicroscopy and how they are currently being applied in industry.

[1] W. C. McCrone, Fusion Methods in Chemical Microscopy, Interscience Publications, New York, 1957. 